\title{
Nanocasting synthesis of graphitized ordered mesoporous carbon using Fe-coated SBA-15 template
}

\author{
Jinjun $\mathrm{Li}^{\mathrm{a}, \mathrm{d}, *}$, Yan Liang ${ }^{\mathrm{a}, \mathrm{b}}$, Baojuan Dou ${ }^{\mathrm{a}}$, Chunyan Ma ${ }^{\mathrm{a}}$, Renjie Lu ${ }^{\mathrm{a}}$, Zhengping Hao ${ }^{\mathrm{a}, *}$, Qiang Xie ${ }^{\mathrm{b}}$, \\ Zhiqiang Luan ${ }^{\mathrm{c}}$, Kai $\mathrm{Li}^{\mathrm{c}}$ \\ ${ }^{a}$ Research Center for Eco-Environmental Sciences, Chinese Academy of Sciences, Beijing 100085, PR China \\ ${ }^{\mathrm{b}}$ China University of Mining \&' Technology (Beijing), Beijing 100083, PR China \\ ${ }^{\mathrm{c}}$ Research Institute of Chemical Defense, Beijing 100191, PR China \\ ${ }^{\mathrm{d}}$ School of Resources and Environmental Sciences, Hubei Key Laboratory of Biomass-Resources, Wuhan University, Wuhan 430079, PR China
}

\section{H I G H L I G H T S}

- One-pot method produces well-ordered Fe-coated SBA-15.

- Template-catalysis method produces graphitized ordered mesoporous carbon.

- Carbons derived from low-molecular-weight phenolic resin are quite graphitizable.

\section{A R T I C L E I N F O}

\section{Article history:}

Received 18 May 2012

Received in revised form

31 October 2012

Accepted 1 December 2012

\section{Keywords:}

Microporous materials

Nanostructures

Phase transitions

Chemical synthesis

\begin{abstract}
A B S T R A C T
Ordered mesoporous carbons with high porosities and graphitized structures were synthesized by a template-catalysis method using low-molecular-weight phenolic resin as carbon precursor and $\mathrm{Fe}-$ coated SBA-15 as both template and catalyst. The synthesis route involves the following steps: (a) the synthesis of mesoporous Fe-coated SBA-15 through a one-pot method, (b) the infiltration of a lowmolecular-weight phenolic resin into the porosity of the Fe-coated SBA-15, (c) the carbonization and catalytic graphitization of the infiltrated phenolic resin at $900{ }^{\circ} \mathrm{C}$, and (d) the removal of the Fe-coated SBA-15 template. The X-ray diffraction, nitrogen sorption, transmission electron microscopy and scanning electron microscopy were used to characterize the materials, and the results indicate that the prepared carbon materials have well-ordered mesoporous structures replicated from the templates, and graphitized structures can be observed on the carbon frameworks, depending on the iron content in the templates.
\end{abstract}

(C) 2012 Elsevier B.V. All rights reserved.

\section{Introduction}

Ordered mesoporous carbons have been of great research interest in recent years because these materials which have high surface areas, tunable mesopore sizes and ordered pore structures are highly attractive for applications as adsorbents, catalyst supports, electrode materials, and so on. In particular, mesoporous carbons with graphitized structures which exhibit high electronic conductivity and improved oxidation resistance appeared to be more promising in energy-related and environmental applications

\footnotetext{
* Corresponding authors. Research Center for Eco-Environmental Sciences, Chinese Academy of Sciences, Beijing 100085, PR China. Tel.: +86 10 62849194; fax: +861062923564.

E-mail addresses: ljjcbacademy@163.com (J. Li), zpinghao@rcees.ac.cn (Z. Hao).
}

[1-6]. Nanocasting, which generally uses mesostructured silicas as hard template, is widely adopted to synthesize ordered mesoporous carbons because it affords a favorable porosity control and good structural ordering of the products [7]. To obtain graphitized mesoporous carbons (GMCs), some additional steps are generally required to achieve graphitization transformation of amorphous carbons. Thermal transformation is a traditional method for the production of graphitized carbons, it needs extremely high temperatures of more than $2200{ }^{\circ} \mathrm{C}$ [8-11], and are likely to cause collapse of mesopores and loss of surface areas. In order to obtain graphitized carbons at relatively low temperatures, some researchers employed special organics as carbon precursors [1217], and it was found that mesophase pitch or some other fused aromatics were quite graphitizable and were likely to transform to graphitized carbons at less than $1000^{\circ} \mathrm{C}$. Another method to obtain graphitized mesoporous carbons at low temperatures is catalytic 
graphitization [3,18-20], and the mostly used metal catalysts include nickel, iron, manganese and cobalt. The catalytic graphitization method generally involves two high-temperature thermal treatments: the first one is carbonization to obtain ordered mesoporous carbons, and the second one is graphitization of the obtained ordered mesoporous carbons at $700-1000{ }^{\circ} \mathrm{C}$ after impregnating metal salts of catalysts on them. Recently, a one-step hard-template method was reported by Lee et al. who used metal phthalocyanines containing cobalt, nickel, manganese or iron in the molecules as carbon precursors [21], when the phthalocyanines infiltrated in mesostructured silicas were carbonized at $900^{\circ} \mathrm{C}$, the self-contained metals catalyzed the graphitization transformation at the same time.

In a previous work, Sevilla and Fuertes synthesized graphitic porous carbons by using a silica xerogel as template and a phenolic resin as carbon precursor, and they introduced catalysts to perform catalytic graphitization after the carbonization of the resin and the removal of silica template [22]. In this paper, we will introduce a one-step nanocasting for the synthesis of ordered mesoporous graphitized carbons with high porosities at a relatively low temperature. Fe-coated SBA-15 with various Fe contents were employed as both templates and catalysts, and common soluble phenolic resin was used as carbon precursors. Characterizations indicate that graphitized mesoporous carbons can be directly replicated from Fe-coated SBA-15, and this can be a relatively easy method for the production of ordered graphitized mesoporous carbons.

\section{Experimental}

\subsection{Chemicals}

Poly (propylene oxide)-block-poly (ethylene oxide)-block-poly (propylene oxide) triblock copolymer P123 $\left(M_{\mathrm{w}}=5800\right.$, $\mathrm{EO}_{20} \mathrm{PO}_{70} \mathrm{EO}_{20}$ ) was provided by Aldrich. Tetraethylorthosilicate (TEOS) was provided by Tianjin Chemical Reagent Company. Iron nitrate (98.5\%) and formaldehyde (37.0-40.0\% aqueous solution) were from Shantou Xilong Chemical Factory. Phenol, hydrochloric acid (36.0-38.0\%), sodium hydroxide (96.0\%), sucrose and ethanol (99.7\%) were from Beijing Chemical Reagent Company. All chemicals were used as received without any further purification.

\subsection{Sample preparation}

\subsubsection{Synthesis of the Fe-coated SBA-15 (FeMS)}

Fe-coated mesoporous silica SBA-15 (FeMS) was synthesized by a one-pot method proposed by Zhu et al. [23], who suggested that such a method could produce SBA-15 with homogeneously dispersed iron oxides on the pore wall framework. In a typical procedure, $4 \mathrm{~g}$ triblock copolymer P123 and certain amount of ferric nitrate were dissolved in $150 \mathrm{~g} 1.6 \mathrm{M} \mathrm{HCl}$, then $8.5 \mathrm{~g}$ TEOS was added under stirring at $40{ }^{\circ} \mathrm{C}$. The molar composition of the mixture was 1TEOS:0.02P123:pFe:6HCl: $192 \mathrm{H}_{2} \mathrm{O}$. After being stirred for $24 \mathrm{~h}$ at $40^{\circ} \mathrm{C}$, the resultant mixture was aged at $100{ }^{\circ} \mathrm{C}$ for $24 \mathrm{~h}$ in an autoclave under static condition. Subsequently, the water was evaporated with stirring at $80^{\circ} \mathrm{C}$ and the solid was further dried at $80^{\circ} \mathrm{C}$ overnight. Finally, the samples were calcined at $550^{\circ} \mathrm{C}$ for $6 \mathrm{~h}$ to remove template and to form the Fe-coated SBA-15 named FeMS- $p$ ( $p$ for the molar ratio $\mathrm{Fe} / \mathrm{Si}$ ).

\subsubsection{Synthesis of phenolic resin precursors}

A low-molecular-weight phenolic resin was prepared from phenol and formaldehyde in a base-catalyzed process [24]. In a typical procedure, $2 \mathrm{~g}$ phenol was melted at $40-42{ }^{\circ} \mathrm{C}$ in a flask and mixed with $0.43 \mathrm{~g} 20 \mathrm{wt} \%$ sodium hydroxide aqueous solution under stirring. After $10 \mathrm{~min}, 3.44 \mathrm{~g}$ formaldehyde (37 wt\%) was added dropwise below $50{ }^{\circ} \mathrm{C}$. The mixture was cooled to room temperature after stirring for $1 \mathrm{~h}$ at $70-75^{\circ} \mathrm{C}$, the $\mathrm{pH}$ was adjusted with $0.6 \mathrm{M}$ hydrochloric acid solution until it reached a value of $\sim 7.0$, and then water was removed by vacuum evaporation below $50{ }^{\circ} \mathrm{C}$. The obtained phenolic resin was dissolved in ethanol, separated from sodium chloride by centrifugation and then dried by vacuum evaporation below $50{ }^{\circ} \mathrm{C}$. The molar ratio of phenol/ formaldehyde/ $\mathrm{NaOH}$ was $1: 2: 0.1$. It was reported that the average molecular weight of such prepared phenolic resin precursors was smaller than 500 [24].

\subsubsection{Synthesis of ordered mesoporous graphitized carbons}

The phenolic resin was infiltrated into FeMS- $p$ by an impregnation method. In a typical synthesis, $0.6 \mathrm{~g}$ phenolic resin was dissolved in $2 \mathrm{~g}$ ethanol and the obtained solution was mixed with $0.5 \mathrm{~g}$ FeMS- $p$ under stirring, after the ethanol has been evaporated at room temperature, the mixture was heated at $80^{\circ} \mathrm{C}$ for $10 \mathrm{~h}$ and subsequently at $160{ }^{\circ} \mathrm{C}$ for another $10 \mathrm{~h}$. The resultant powders were put into the ethanolic solution of phenolic resin again for another impregnation to load more phenolic resin into FeMS- $p$ ( $0.4 \mathrm{~g}$ phenolic resin, $2 \mathrm{~g}$ ethanol). After retreated at 80 and $160{ }^{\circ} \mathrm{C}$ as before, the resultant solid was carbonized at $900{ }^{\circ} \mathrm{C}$ in a tube furnace under nitrogen flow for $2 \mathrm{~h}$, and then treated in $2 \mathrm{M}$ sodium hydroxide solution at $80{ }^{\circ} \mathrm{C}$ under stirring to remove the silica frameworks, and finally treated in concentrated hydrochloric acid (36.0-38.0\%) overnight to remove the iron, forming the final carbon product named SGC- $p$.

For comparison purpose, sucrose, which used to be employed to prepare ordered mesoporous carbons, was also used as a carbon precursor, and the synthesis procedure is similar to the literatures [7,25]. $0.625 \mathrm{~g}$ sucrose and $0.07 \mathrm{~g} \mathrm{H}_{2} \mathrm{SO}_{4}$ were dissolved in $2.5 \mathrm{~g} \mathrm{H}_{2} \mathrm{O}$ to form the sucrose solution and the sucrose solution was impregnated onto $0.5 \mathrm{~g}$ FeMS- $p$ under stirring. The mixture was cured at $80^{\circ} \mathrm{C}$ for $10 \mathrm{~h}$ and subsequently at $160^{\circ} \mathrm{C}$ for another $10 \mathrm{~h}$. After that the resultant powder was impregnated with the sucrose solution ( $0.375 \mathrm{~g}$ sucrose, $0.04 \mathrm{~g} \mathrm{H}_{2} \mathrm{SO}_{4}$ and $2.5 \mathrm{~g} \mathrm{H}_{2} \mathrm{O}$ ) for the second time, and retreated at 80 and $160{ }^{\circ} \mathrm{C}$ again, the resultant powder was carbonized at $900{ }^{\circ} \mathrm{C}$ in a tube furnace under nitrogen flow for $2 \mathrm{~h}$, and then the silica framework and iron was removed in the same way as mentioned above, forming the product named Suc-SGC- $p$.

\subsection{Characterizations}

X-ray diffraction (XRD) patterns were recorded on PANalytical X'Per PRO MPD using a Cu-K $\alpha(\lambda=1.540598 \AA)$ radiation. High resolution transmission electron microscopy (HRTEM) images were taken on a JEOL 2011 at an acceleration voltage of $200 \mathrm{kV}$. Scanning electron microscopy (SEM) images were taken with a Hitachi S$3000 \mathrm{~N}$ electron microscopy operating at $5 \mathrm{kV}$ accelerating voltage, and a thin gold film was sprayed on the sample before characterization. Nitrogen adsorption/desorption isotherms were obtained at liquid nitrogen temperature on a NOVA1200 gas sorption analyzer. The samples were evacuated at $573 \mathrm{~K}$ for $7 \mathrm{~h}$ before measurements. The BET surface areas were calculated using experimental points at a relative pressure of $P / P_{0}=0.05-0.25$. Total pore volumes were estimated from the nitrogen amount adsorbed at a relative pressure $\left(P / P_{0}\right)$ of about 0.99 . The pore size distribution curves were derived from the adsorption branch of the isotherms using Barrett-Joyner-Halenda (BJH) method.

\section{Results and discussion}

Fig. 1 shows the XRD patterns of the host templates and the carbon replicas. The template FeMS-0.01, 0.02 and 0.04 show three 

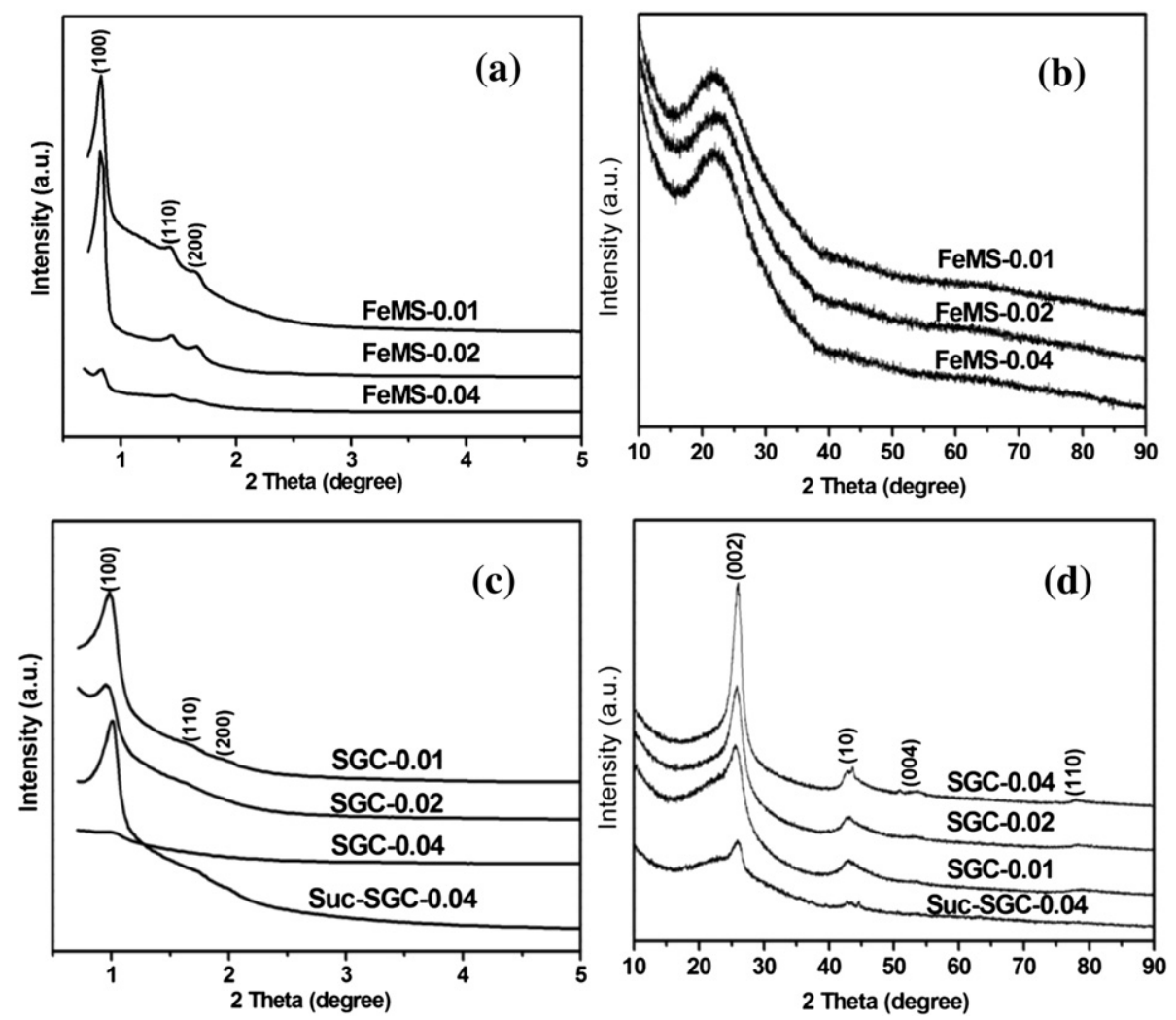

Fig. 1. XRD patterns of (a, b) FeMS templates and (c, d) carbon replicas.

well-resolved diffraction peaks on their small-angle XRD patterns (Fig. 1a), which is attributed to the (100), (110) and (200) diffraction of hexagonal mesostructure, indicating that these samples possess typical ordered structure of SBA-15 [26]. With the increase of Fe content, the intensity of the diffraction peaks decreases, suggesting that more Fe content results in a relatively lower mesostructural ordering of the templates. In Fig. 1b, there is not any crystalline phase associated with iron oxide in the wide-angle XRD patterns of the FeMS samples, which indicates that the Fe species are highly dispersed on the mesoporous framework.

The small-angle XRD patterns of the carbon replicas SGCs also show diffraction peaks which is associated with ordered hexagonal mesostructure (Fig. 1c), indicating that the hexagonal structure of the template was successfully replicated. The wide-angle XRD patterns of the SGCs show four well resolved diffraction peaks at around $25.8,43.1,53.3$ and $78.4^{\circ}$ (Fig. $1 \mathrm{~d}$ ), which can be assigned to the $002,10,004$ and 110 reflections of graphitized carbons [19,27], indicating that the SGCs have graphitized structures. The interlayer distance $\left(d_{002}\right)$ between the graphene planes were calculated based on the Bragg formula and the results are displayed in Table 1. It is known that the ideal graphite has an interlayer distance $\left(d_{002}\right)$ of $3.354 \AA[19,23]$, the $d_{002}$ values of the SGCs are a little larger than that of the ideal graphite, indicating that these samples have somewhat turbostratic structures. It is well documented that Fe is an effective catalyst for the graphitization transformation of amorphous carbonaceous materials, and the catalytic graphitization process involves the formation of iron carbide at high temperatures and the subsequent precipitation of graphite from the carbide species [27]. In this work, the template materials have well dispersed iron species on their pore walls [23], and these iron species can induce the graphitization transformation of the carbon materials derived from the infiltrated phenolic resin in the mesopore channels. The catalytic graphitization that occurred in mesoporous silicas was also observed by Lee et al. [21], who used metal-containing phthalocyanines as carbon precursors to synthesize ordered mesoporous carbons.

Fig. $1 \mathrm{~d}$ indicates that the Fe content in the silica templates is crucial to the graphitization of the final mesoporous carbons. With increasing the molar ratio $\mathrm{Fe} / \mathrm{Si}$ from 0.01 to 0.04 , the intensity of the (002) peak gradually increases, indicating that more Fe content leads to larger ordered domain of graphitized carbon, at the same time, the $d_{002}$ value decreases gradually, getting closer to typical ideal graphite $(3.354 \AA$ ) [23]. A higher Fe content is favorable for producing better graphitized structure because $\mathrm{Fe}$ is a catalyst for the graphitization. However, more iron tends to decrease the mesostructural ordering of the template as mentioned above, therefore resulting in decrease in the mesostructural ordering of the replicated SGCs as well (Fig. 1c).

Sucrose is the mostly used carbon precursor for the synthesis of ordered mesoporous carbons in literatures [25]. In this work, sucrose was also used as carbon precursor for comparison purpose. Although the small-angle XRD pattern of Suc-SGC-0.04 sample

Table 1

Physical and textural properties of the templates and the carbon replicas.

\begin{tabular}{lllll}
\hline Sample & $S_{\text {BET }}\left(\mathrm{m}^{2} \mathrm{~g}^{-1}\right)$ & $V_{\text {total }}\left(\mathrm{cm}^{3} \mathrm{~g}^{-1}\right)$ & $D^{\mathrm{a}}(\mathrm{nm})$ & $d_{002}^{\mathrm{b}}(\AA)$ \\
\hline SGC-0.01 & 469 & 0.43 & 4.63 & 3.475 \\
SGC-0.02 & 670 & 0.62 & 4.27 & 3.451 \\
SGC-0.04 & 360 & 0.54 & 3.68 & 3.421 \\
Suc-SGC-0.04 & 471 & 0.79 & $<2$ & 3.444 \\
FeMS-0.01 & 816 & 1.05 & 9.69 & - \\
FeMS-0.02 & 749 & 1.12 & 9.70 & - \\
FeMS-0.04 & 565 & 0.95 & 9.70 & - \\
\hline
\end{tabular}

${ }^{a}$ Pore diameters derived from the adsorption branches of nitrogen isotherms by using the BJH model.

b $d_{002}$ interlayer spacing values evaluated from the wide-angle XRD patterns using Bragg formula. 
synthesized by using sucrose as carbon source shows good mesostructural ordering (Fig. 1c), its wide-angle XRD pattern shows much lower diffraction peaks compared to that of SGCs (Fig. 1d), which indicates that it has a relatively lower graphitization degree. A previous study revealed that aromatic hydrocarbons afford carbon derivatives with improved graphitic character [16]. In this work, the abundant aromatic rings in the phenolic resin might also be more favorable for the graphitization transformation than sucrose.
Fig. 2 shows the transmission electron microscope (TEM) and scanning electron microscope (SEM) images of the templates and the carbon replicas. The TEM images of FeMS templates show wellordered hexagonal mesostructures (Fig. 2a-c) which further verifies that SBA-15 doped with Fe maintained its mesostructural ordering. The high-resolution transmission electron microscopy (HRTEM) images of the prepared SGC samples (Fig. 2d-i) show apparent ordered mesoporous structure with hexagonal symmetry,
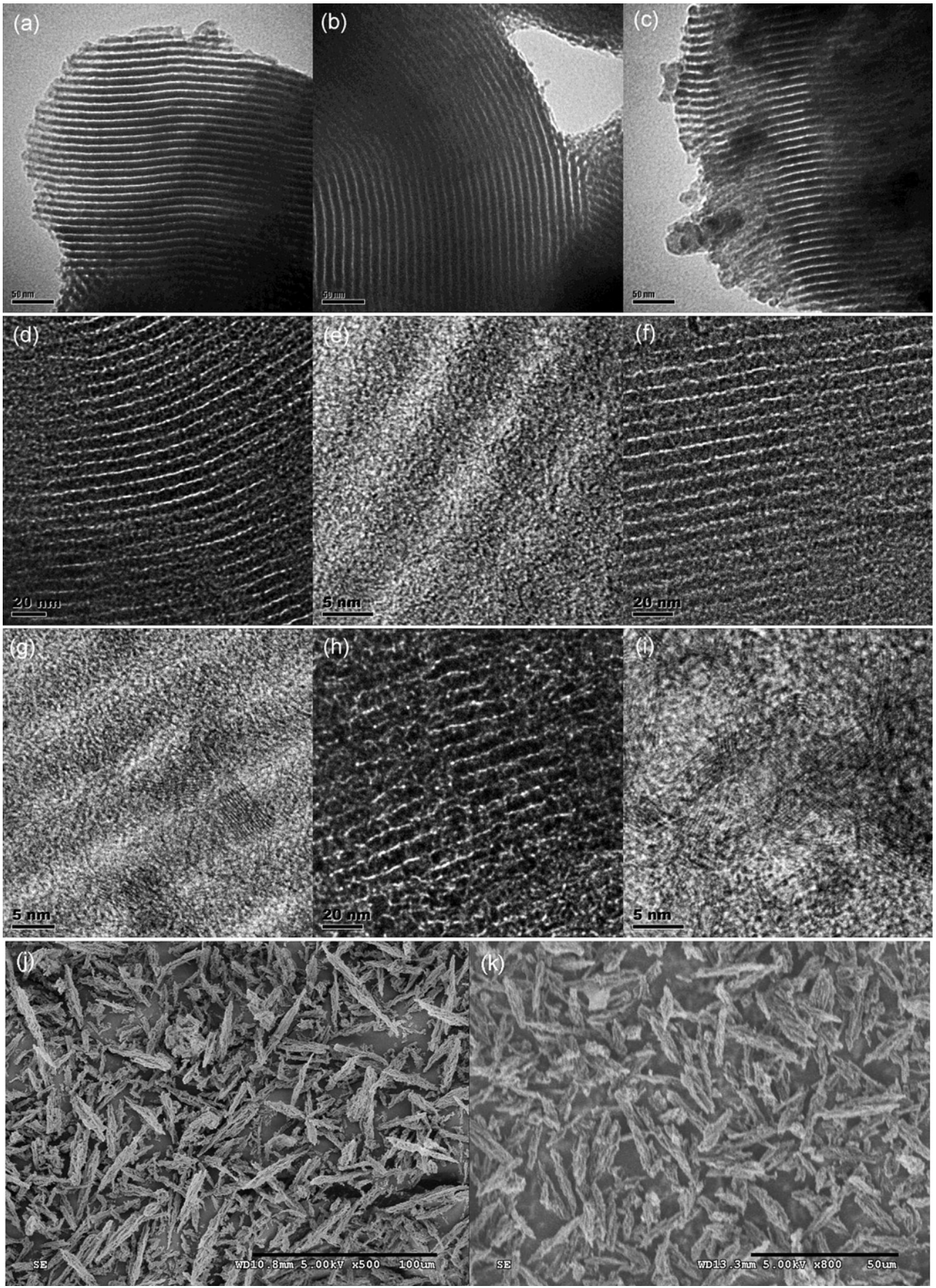

Fig. 2. TEM images of (a) FeMSn-0.01, (b) FeMSn-0.02, (c) FeMSn-0.04, HRTEM images of (d, e) SGC-0.01, (f, g) SGC-0.02, (h, i) SGC-0.04, and SEM images of (j) FeMSn-0.02, (k) SGC0.02 . 

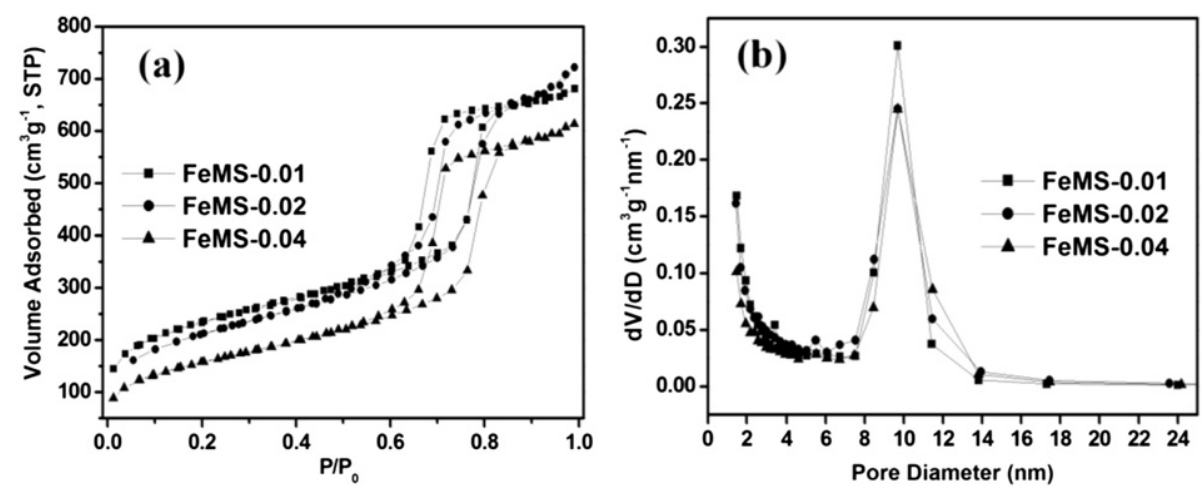

Fig. 3. (a) Nitrogen adsorption-desorption isotherms and (b) BJH pore size distribution curves of FeMS-0.01, 0.02 and 0.04 .

which also confirms that the SGC samples are good inverse replicas of their templates. Fig. 2e, $g$ and i shows the high-magnification HRTEM images of SGC samples, and stacking graphene layers can be directly observed on the mesopore wall, and it is apparent that the prepared carbons show more graphitized domains with the increase of Fe content in templates, which is also in consistence with the XRD results. The scanning electron microscopy (SEM) images of FeMS-0.02 and its replica SGC-0.02 is shown in Fig. 2k and $\mathrm{j}$, respectively. It revealed that both samples consist of wheatlike particles, and the similar morphology can also indicate a faithful replication.

Fig. 3 shows the nitrogen adsorption-desorption isotherms and the BJH pore size distribution curves for FeMS templates. Typical type IV isotherms with $\mathrm{H} 1$ hysteresis loop which is typical for mesoporous materials are observed for the FeMS templates (Fig. 3a) [26]. Sharp capillary condensation of nitrogen occurred at relative pressure of $0.6-0.8$, indicating the uniformity of the mesopores. With the increase of the molar ratio $\mathrm{Fe} / \mathrm{Si}$, the position of the capillary condensation tends to shift to higher relative pressure, suggesting the increase of the mesopore size, which is clearly reflected by the pore size distribution profile (Fig. 3b). Physical and textural properties of FeMS templates with different Fe/Si molar ratios are listed in Table 1 . It is noticeable that these template materials have highly porous structures, and the specific surface areas decrease with the increase of $\mathrm{Fe} / \mathrm{Si}$ molar ratio.

Fig. 4 shows the nitrogen adsorption-desorption isotherms and the BJH pore size distribution curves of SGCs. Sample SGC-0.01 exhibited a type IV isotherm with a relatively steep condensation step, which is characteristic of mesoporous solids with relatively uniform mesopores [28]. And the pore size distribution profile of
SGC-0.01 indicates that most of its porosity is distributed from 2.9 to $6.1 \mathrm{~nm}$ with a maximum distribution at $4.6 \mathrm{~nm}$. The relatively narrow pore size distribution might arise from the uniformity of the template framework. Nevertheless, SGC-0.02 and SGC-0.04 samples exhibit two-step isotherms (Fig. 4a), and they have broader pore size distribution than SGC-0.01 (Fig. 4b), and apart from the main distribution from 2 to $6 \mathrm{~nm}$, quite a lot of porosities are distributed in a wide range with diameters larger than $6 \mathrm{~nm}$. The broadening of the pore size distribution of SGC- 0.02 and SGC0.04 can be explained in terms of the Fe content. During the carbonization of the phenolic resin infiltrated in the FeMS template, the phenolic resin would transform into carbon rods confined in the cylindrical mesopores of FeMS, and the iron oxide species on the pore wall of FeMS could be reduced by carbon to iron metal, which catalyze the graphitization of the carbon rods. With a high Fe content, the iron metal tend to agglomerate into larger particles at improved temperatures, and the iron particles might immigrate into the carbon rods during the catalytic graphitization reaction, and therefore the final acid digesting treatment to remove iron particles would leave secondary porosities on the carbon rods. A higher Fe content in FeMS-0.04 would cause bigger iron particles, therefore leading to larger secondary porosities on the final products. Actually in the HRTEM images of SGC-0.04 (Fig. 2h), the splitting of the carbon rods can be easily observed, which can be caused by the removal of very large iron particles intercalated in the carbon rods. The derived textural property data are listed in Table 1. Although the graphitization process tends to reduce porosities significantly because of the crystallization of carbon atoms, the SGCs have high specific surface areas ranging from 469 to $670 \mathrm{~m}^{2} \mathrm{~g}^{-1}$, which can be attributed to the hard template effect.
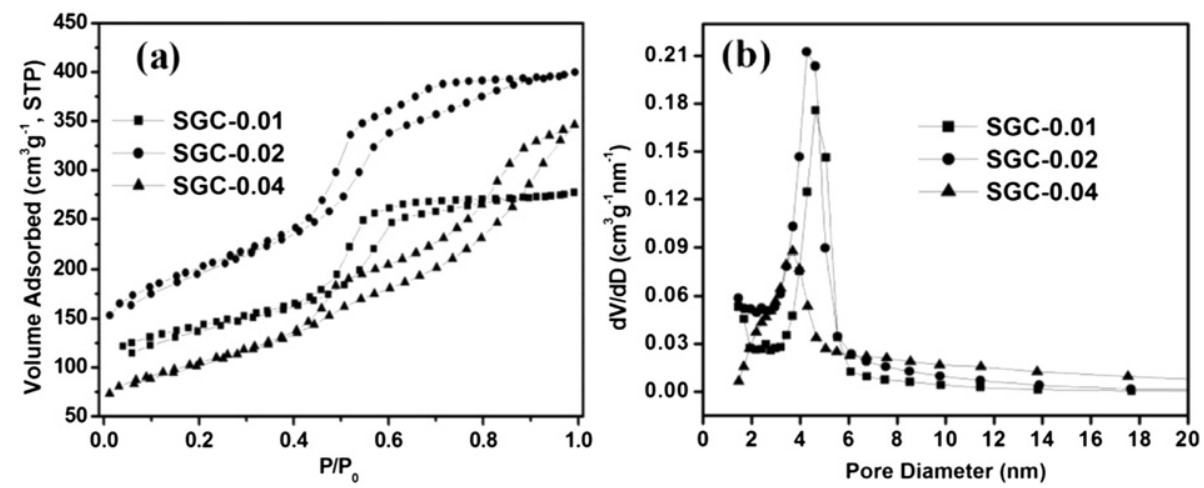

Fig. 4. (a) Nitrogen adsorption-desorption isotherms and (b) BJH pore size distribution curves of SGC-0.01, 0.02 and 0.04 . 


\section{Conclusion}

Ordered mesoporous carbons with graphitized framework can be synthesized using Fe-coated SBA-15 as both template and graphitization catalyst, and low-molecular-weight phenolic resin as carbon precursor. The iron in the mesoporous silica template can catalyze the graphitization transformation of carbon precursors during the carbonization and the mesoporous silicas can template the formation of highly ordered mesostructures of the products.

\section{Acknowledgment}

This work was funded by the National Science Foundation of China (50978248, 20807050, 20725723).

\section{References}

[1] C.H. Huang, R.A. Doong, D. Gu, D.Y. Zhao, Carbon 49 (2011) 3055-3064

[2] F.B. Su, J.H. Zeng, X.Y. Bao, Y.S. Yu, J.Y. Lee, X.S. Zhao, Chem. Mater. 17 (2005) 3960-3967.

[3] A.M. Wang, J.W. Ren, B.F. Shi, G.Z. Lu, Y.Q. Wang, Microporous Mesoporous Mater. 151 (2012) 287-292.

[4] Z.H. Hou, F.Y. Zeng, B.H. He, W. Tao, C.Y. Ge, Y.F. Kuang, J.H. Zeng, Mater. Lett 65 (2011) 897-900.

[5] X.Z. Cui, J.L. Shi, L.X. Zhang, M.L. Ruan, J.H. Gao, Carbon 47 (2009) 186-194.

[6] F.B. Su, X.S. Zhao, Y. Wang, J.H. Zeng, Z.C. Zhou, J.Y. Lee, J. Phys. Chem. B 109 (2005) 20200-20206.

[7] S. Jun, S.H. Joo, R. Ryoo, M. Kruk, M. Jaroniec, Z. Liu, T. Ohsuna, O. Terasaki, J. Am. Chem. Soc. 122 (2000) 10712-10713.

[8] A.B. Fuertes, S. Alvarez, Carbon 42 (2004) 3049-3055.
[9] K.P. Gierszal, M. Jaroniec, T.W. Kim, J. Kim, R. Ryoo, New J. Chem. 32 (2008) 981-993.

[10] M. Kruk, K.M. Kohlhaas, B. Dufour, E.B. Celer, M. Jaroniec, K. Matyjaszewski, R.S. Ruoff, T. Kowalewski, Microporous Mesoporous Mater. 102 (2007) $178-187$.

[11] S.B. Yoon, G.S. Chai, S.K. Kang, J.S. Yu, K.P. Gierszal, M. Jaroniec, J. Am. Chem. Soc. 127 (2005) 4188-4189.

[12] L. Cao, M. Kruk, Adsorption 16 (2010) 465-472.

[13] H.F. Yang, Y. Yan, Y. Liu, F.Q. Zhang, R.Y. Zhang, Y. Meng, M. Li, S.H. Xie, B. Tu, D.Y. Zhao, J. Phys. Chem. B 108 (2004) 17320-17328

[14] T.W. Kim, I.S. Park, R. Ryoo, Angew. Chem. Int. Ed. 42 (2003) 4375-4379.

[15] N. Gokulakrishnan, N. Kania, B. Leger, C. Lancelot, D. Grosso, E. Monflier, A. Ponchel, Carbon 49 (2011) 1290-1298.

[16] C.H. Kim, D.K. Lee, T.J. Pinnavaia, Langmuir 20 (2004) 5157-5159.

[17] G. Gupta, D.A. Slanac, P. Kumar, J.D. Wiggins-Camacho, J. Kim, R. Ryoo, K.J. Stevenson, K.P. Johnston, J. Phys. Chem. C 114 (2010) 10796-10805.

[18] W.J. Gao, Y. Wan, Y.Q. Dou, D.Y. Zhao, Adv. Energy Mater. 1 (2011) 115-123.

[19] S.J. Yi, Z. Fan, C. Wu, J.H. Chen, Carbon 46 (2008) 378-380.

[20] J. Choma, K. Jedynak, M. Marszewski, M. Jaroniec, Appl. Surf. Sci. 258 (2012) 3763-3770.

[21] K.T. Lee, X.L. Ji, M. Rault, L.F. Nazar, Angew. Chem. Int. Ed. 48 (2009) 56615665.

[22] M. Sevilla, A.B. Fuertes, Carbon 44 (2006) 468-474

[23] Y.M. Wang, Z.Y. Wu, Y.L. Wei, J.H. Zhu, Microporous Mesoporous Mater. 84 (2005) 127-136

[24] Y. Meng, D. Gu, F.Q. Zhang, Y.F. Shi, L. Cheng, D. Feng, Z.X. Wu, Z.X. Chen, Y. Wan, A. Stein, D.Y. Zhao, Chem. Mater. 18 (2006) 4447-4464.

[25] Y.P. Zhai, Y. Wan, Y. Cheng, Y.F. Shi, F.Q. Zhang, B. Tu, D.Y. Zhao, J. Porous Mater. 15 (2008) 601-611.

[26] D. Zhao, Q. Huo, J. Feng, B.F. Chmelka, G.D. Stucky, J. Am. Chem. Soc. 120 (1998) 6024-6036.

[27] Y.G. Wang, Y. Korai, I. Mochida, K. Nagayama, H. Hatano, N. Fukuda, Carbon 39 (2001) 1627-1634.

[28] M. Kruk, M. Jaroniec, Chem. Mater. 13 (2001) 3169-3183. 